\title{
DGAT1-dependent triacylglycerol storage by macrophages protects mice from diet- induced insulin resistance and inflammation
}

\author{
Suneil K. Koliwad, ${ }^{1,2,3,4}$ Ryan S. Streeper, ${ }^{1}$ Mara Monetti, ${ }^{1}$ Ivo Cornelissen, ${ }^{3}$ Liana Chan, ${ }^{1}$ \\ Koji Terayama, ${ }^{5}$ Stephen Naylor, ${ }^{6}$ Meghana Rao, ${ }^{1}$ Brian Hubbard,7 and Robert V. Farese Jr. ${ }^{1,2,3,4,8}$

\begin{abstract}
${ }^{1}$ Gladstone Institute of Cardiovascular Disease, San Francisco, California. ${ }^{2}$ Diabetes Center, ${ }^{3}$ Cardiovascular Research Institute, and ${ }^{4}$ Department of Medicine, UCSF, San Francisco, California. ${ }^{5}$ Daiichi Sankyo Co. Ltd., Tokyo, Japan. ${ }^{6}$ Department of Physiology, UCSF.
\end{abstract} \\ ${ }^{7}$ Merck Research Laboratories, Rahway, New Jersey. ${ }^{8}$ Department of Biochemistry and Biophysics, UCSF.
}

\begin{abstract}
Diet-induced obesity (DIO) leads to inflammatory activation of macrophages in white adipose tissue (WAT) and subsequently to insulin resistance. PPAR $\gamma$ agonists are antidiabetic agents known to suppress inflammatory macrophage activation and to induce expression of the triacylglycerol (TG) synthesis enzyme acyl CoA: diacylglycerol acyltransferase 1 (DGAT1) in WAT and in adipocytes. Here, we investigated in mice the relationship between macrophage lipid storage capacity and DIO-associated inflammatory macrophage activation. Mice overexpressing DGAT1 in both macrophages and adipocytes (referred to herein as aP2-Dgat1 mice) were more prone to DIO but were protected against inflammatory macrophage activation, macrophage accumulation in WAT, systemic inflammation, and insulin resistance. To assess the contribution of macrophage DGAT1 expression to this phenotype, we transplanted wild-type mice with aP2-Dgat1 BM. These mice developed DIO similar to that of control mice but retained the protection from WAT inflammation and insulin resistance seen in aP2-Dgat1 mice. In isolated macrophages, Dgat $1 \mathrm{mRNA}$ levels correlated directly with TG storage capacity and inversely with inflammatory activation by saturated fatty acids (FAs). Moreover, PPAR $\gamma$ agonists increased macrophage Dgat $1 \mathrm{mRNA}$ levels, and the protective effects of these agonists against FA-induced inflammatory macrophage activation were absent in macrophages isolated from Dgat1-null mice. Thus, increasing DGAT1 expression in murine macrophages increases their capacity for TG storage, protects against FA-induced inflammatory activation, and is sufficient to reduce the inflammatory and metabolic consequences of DIO.
\end{abstract}

\section{Introduction}

Chronic diet-induced obesity (DIO) promotes infiltration of the white adipose tissue (WAT) by monocyte-derived phagocytic and antigen-presenting cells that include macrophages and dendritic cells. These macrophages accumulate in crown-like structures (CLS) around dying or apoptotic adipocytes and change their overall polarity from a relatively antiinflammatory (M2) activation state to a more inflammatory (M1) state (1-5). The M1 activation of macrophages in WAT is linked to chronically elevated serum levels of inflammatory cytokines, such as TNF- $\alpha$, that are key to the development of insulin resistance, diabetes, and atherosclerosis (6-9).

Determining the molecular mechanisms that trigger $\mathrm{M} 1$ activation of macrophages in WAT during DIO may offer insights into the metabolic consequences of obesity. Recent evidence points to the importance of saturated fatty acids (FAs), which are systemically elevated in chronic DIO $(10,11)$ and stimulate M1 activation of macrophages and dendritic cells in culture (12-15). This stimulation is mediated, at least in part, by signaling through TLR2 and TLR4 and the associated activation of JNK (13-17). However, M1 activation may also be influenced by the levels of intracellular FAs, which regulate a wide variety of signaling pathways (18-22). These intracellular FAs exist in different pools, including free FAs, FA acyl CoAs, and inert FA moieties that are incorporated into glycerolipids, such as triacylglycerols (TGs) (23). The capacity of macrophages to incorporate FAs into TGs could thus serve to modulate the potential for M1 activation.

Conflict of interest: The authors have declared that no conflict of interest exists. Citation for this article: J Clin Invest. 2010;120(3):756-767. doi:10.1172/JCI36066.
Synthesis of TG involves acyl CoA:diacylglycerol acyltransferase (DGAT) enzymes, which catalyze a reaction with diacylglycerol and FA acyl CoA substrates (23). Cellular TG storage is directly correlated with levels of DGAT activity. For example, overexpression of DGAT enzymes in transgenic mice is sufficient to increase cellular TG storage in WAT, skeletal muscle, or liver in a tissue-specific manner (24-26). Moreover, DGAT1 helps to protect cultured cells from the toxic effects of excess FAs (27). Interestingly, DGAT1 expression in adipocytes and WAT is upregulated by PPAR $\gamma$ activation (28-30), and PPAR $\gamma$ activation protects macrophages against M1 activation in vitro and in vivo (13, 31-33). Given these findings, we hypothesized that DGAT1 expression in macrophages may modulate M1 activation by FAs.

In the current study, we examined this hypothesis by performing a series of studies in genetically modified mice to determine how increasing DGAT1 expression in the macrophage compartment affects the response to a chronically high-fat diet. We also examined primary macrophages from genetically modified mice to determine whether the level of DGAT1 expression modulates FA- and cytokineinduced M1 activation in a cell-autonomous manner. Finally, we determined whether DGAT1 is regulated by PPAR $\gamma$ agonists in macrophages and whether DGAT1 is required for the antiinflammatory effects of PPAR $\gamma$ agonists on FA-induced M1 activation.

\section{Results}

aP2-Dgat1 mice have increased Dgat1 $m R N A$ levels in both adipocytes and macrophages. We previously showed that transgenic C57BL/6 mice overexpressing murine Dgat1 under the control of the FA-binding 


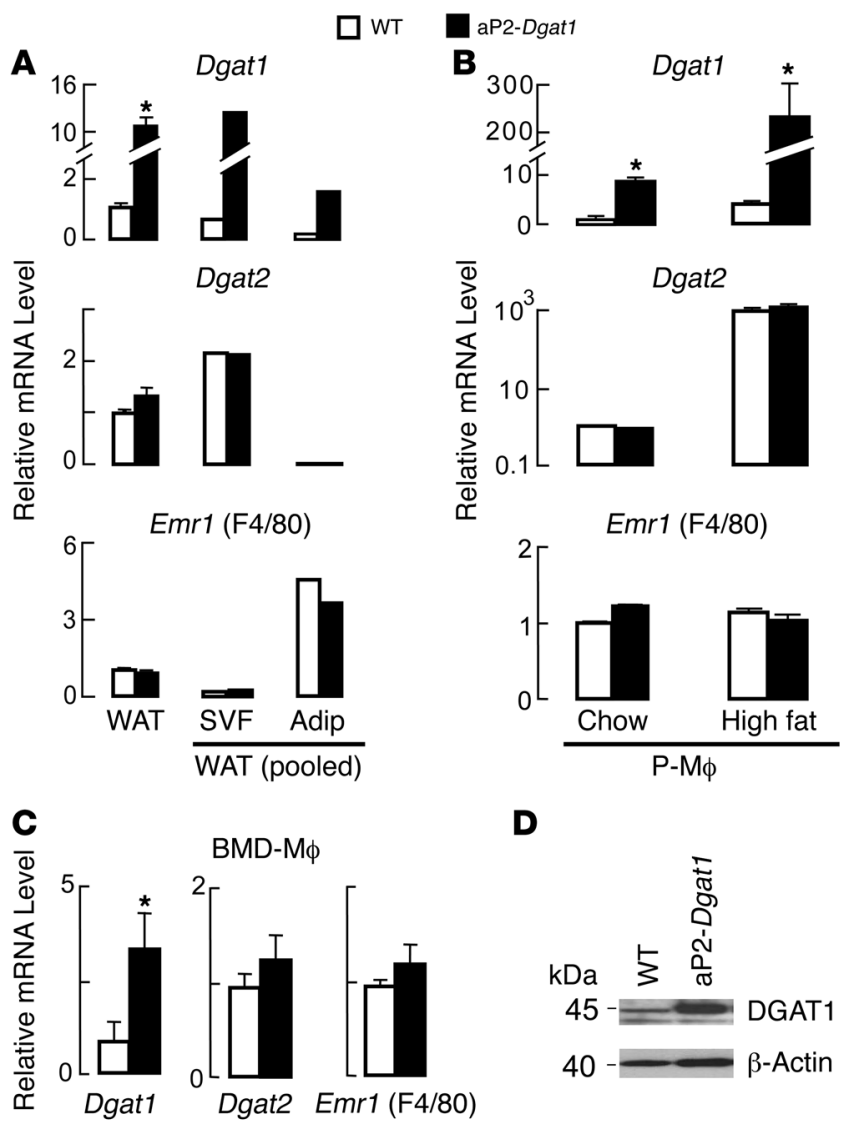

protein 4 (aP2) promoter (aP2-Dgat1) have increased Dgat1 mRNA levels in WAT (24). However, the aP2 promoter can drive gene expression in both adipocytes and monocyte-derived macrophages and dendritic cells $(34,35)$. We therefore examined the contribution of these cell types to increased Dgat1 mRNA levels in aP2-Dgat1 WAT. We harvested the epididymal fat pads from mice, keeping 1 fat pad intact and separating the other to yield adipocytes and a macrophage-containing stromal-vascular fraction (SVF). We then analyzed mRNA levels in the whole WAT, adipocytes, and SVF by quantitative PCR (qPCR) (Figure 1A). Dgat2 mRNA was highly expressed specifically in adipocytes, a cell type rich in DGAT2, while Emr1 (EGF-like module-containing mucin-like receptor 1 or F4/80), a macrophage-expressed gene, was highly expressed in the SVF, verifying the integrity of the adipocyte and macrophage fractions. Although Dgat1 mRNA levels were, as expected, increased $(\sim 10$-fold $)$ in the whole WAT of aP2-Dgat 1 mice, this increase occurred to a similar magnitude in adipocytes and the SVF, suggesting that the transgene is expressed in both adipocytes and monocyte-derived macrophages.

To assess DGAT1 overexpression specifically in macrophages, we measured Dgat1 mRNA levels in peritoneal macrophages (P-M $\phi)$ and BM-derived macrophages (BMD-M $\phi$ ) from aP2-Dgat1 mice. Whereas the mRNA levels of controls Dgat2 and Emr1 were similar in WT and aP2-Dgat1 mice, Dgat1 mRNA levels were increased ( 6-8.5-fold) in the P-M $\phi$ of aP2-Dgat1 mice whether they were fed a chow diet or a 16-week high-fat diet (Figure 1B). Interestingly, mRNA levels of both Dgat 1 and Dgat2 were increased dramatically $(\sim 50$-fold for Dgat1 and $\sim 800$-fold for Dgat 2$)$ in macrophages after high-fat feeding in WT mice (Figure 1B), suggesting both enzymes

\section{Figure 1}

Dgat1 mRNA levels are increased in both adipocytes and macrophages from aP2-Dgat1 mice. (A) Increased Dgat1 mRNA levels in whole WAT and in adipocyte (Adip) and SVF fractions of aP2-Dgat1 mice fed a chow diet. mRNA levels were measured by qPCR and normalized for each gene product to levels in whole WAT for WT $(n=5-6$ per group; ${ }^{*} P<0.05$ ). Samples were pooled for adipocytes and SVF. (B) Increased Dgat1 mRNA levels in P-M $\phi$ of aP2-Dgat1 mice fed a chow or high-fat diet. mRNA levels were measured by qPCR $(n=4-8$ per group; * $P<0.05$ ). Mean Ct values for Dgat1, Dgat2, and Emr1 (F4/80) were 27.6, 30.2, and 25.0, respectively, for WT chow-fed samples and 25.0, 24.0, and 24.9, respectively, for WT high-fat samples. Data for each gene were normalized to WT chow. (C) Increased Dgat1 mRNA levels in BMD-M $\phi$ of aP2-Dgat1 fed a chow diet. mRNA levels were measured by qPCR ( $n=4-8$ per group; $\left.{ }^{*} P<0.05\right)$. (D) Representative immunoblot demonstrating increased DGAT1 protein in BMD-M $\phi$ of aP2-Dgat1 mice.

are involved in the response of macrophages to a high-fat diet. For Dgat1, the increase was further accentuated in the transgenic mice such that mRNA levels were over 200-fold higher than baseline levels. Dgat1 mRNA levels were also increased in BMD-M $\phi$ from aP2-Dgat1 mice (Figure 1C), as were DGAT1 protein levels (Figure 1D).

aP2-Dgat 1 mice are prone to accentuated DIO but protected against its metabolic and inflammatory consequences. To determine the impact of increased Dgat1 mRNA levels in the adipocyte/macrophage compartment on obesity and diet-induced insulin resistance, WT and aP2-Dgat1 mice were fed a chow diet or a high-fat diet for 16 weeks. On either diet, aP2-Dgat1 mice gained more weight than WT mice (Figure 2A), and an analysis of body composition by dual energy X-ray absorptiometry (DEXA) showed increased adiposity (Figure 2B). Glucose tolerance was similar in aP2-Dgat1 and WT mice fed a chow diet (data not shown). However, despite their relative obesity, aP2-Dgat1 mice chronically fed a high-fat diet had less TG accumulation in the skeletal muscle and liver (Figure 2C) and enhanced glucose and insulin tolerance (Figure 2D). aP2-Dgat1 mice with chronic DIO also had lower fasting levels of insulin and glucose and a lower mean value on the homeostasis model assessment of insulin resistance (HOMA-IR) index, indicative of improved insulin sensitivity (Table 1). aP2-Dgat1 mice with chronic DIO displayed other phenotypic features associated with enhanced insulin sensitivity. Serum resistin levels, which increase with DIO and promote insulin resistance (36), were not elevated in aP2-Dgat1 mice, and serum adiponectin levels, which correlate inversely with the degree of DIO and insulin resistance (37), were higher in aP2-Dgat1 mice (Table 1). Additionally, aP2-Dgat1 mice had lower serum TG levels when fed a high-fat diet (Table 1), as reported (24).

Given their improvement in insulin and glucose metabolism, we hypothesized that the aP2-Dgat1 mice would have less dietinduced inflammation in the WAT. In agreement with this, the expression of genes induced by M1 macrophage activation, including Nos2 (iNOS), Itgax (integrin, alpha X [complement component 3 receptor 4 subunit] or CD11c), and those encoding for inflammatory cytokines (Tnfa), and chemokines ( $\mathrm{Ccl} 2$, or monocyte chemoattractant protein-1 [MCP-1]), were reduced in the WAT of aP2-Dgat1 mice chronically fed a high-fat diet (Figure 3A). In contrast, mRNA levels for genes marking the alternative (M2) activation state of macrophages were not different between genotypes (Figure 3A). Taken together, these data indicate that aP2-Dgat1 mice chronically fed a high-fat diet have reduced activation of the M1 inflammatory pathway in the WAT. 

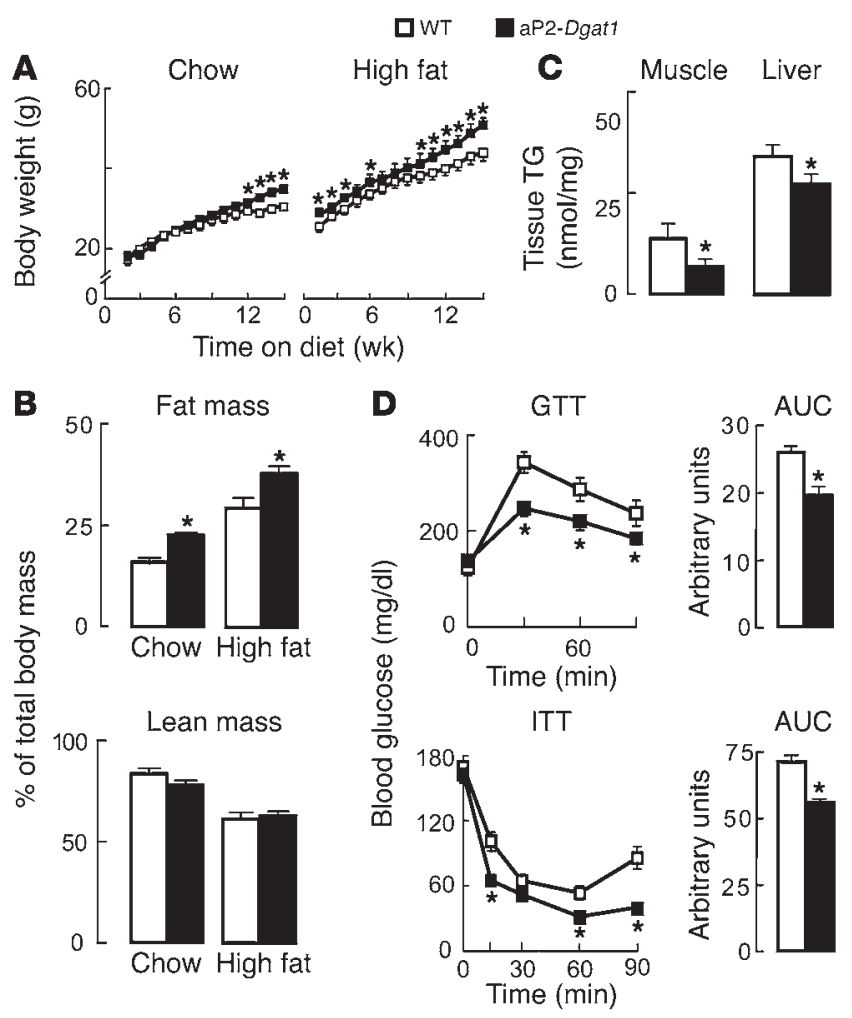

Activated resident WAT macrophages promote inflammation by recruiting more macrophages, which accumulate in CLS around adipocytes on histological sections (1-5). The number of such structures in WAT was reduced by more than 50\% in high-fat-fed aP2-Dgat1 mice (Figure 3B). Thus, DIO in aP2-Dgat1 mice is associated with reductions in both the M1 activation and accumulation of macrophages in the WAT, 2 components of chronic WAT inflammation. These differences were not seen in chow-fed mice, in which CLS were rarely seen in WAT (data not shown). Consistent with decreased recruitment of macrophages into the WAT, we found that aP2-Dgat 1 mice had 20\%-40\% lower levels of the serum proinflammatory factors TNF- $\alpha, \mathrm{MCP}-1$, and plasminogen activator inhibitor-1 (PAI-1) (Figure 3C).

Protection against inflammatory and metabolic consequences of DIO in aP2-Dgat1 mice does not require adiponectin. The WAT-derived hormone adiponectin modulates metabolic and inflammatory parameters in mice (29, 38-42). Because aP2-Dgat1 mice had $60 \%$ more serum adiponectin (Table 1), adiponectin emerged as a potential contributor to the phenotype of aP2-Dgat 1 mice. To address this possibility, we crossed aP2-Dgat1 mice with adiponectin-deficient $\left(\right.$ Adipoq $\left.^{-/}\right)$mice (39) to generate Adipoq ${ }^{-/}$aP2-Dgat1 mice and examined the inflammatory and metabolic responses of these mice to chronic DIO. On a chow diet, Adipoq ${ }^{-1-}$ aP2-Dgat1 mice gained slightly more weight than Adipoq ${ }^{-/-}$mice (Supplemental Figure 1A; supplemental material available online with this article; doi:10.1172/JCI36066DS1).

\section{Figure 2}

aP2-Dgat1 mice are prone to obesity but protected against its metabolic consequences. (A) Growth curves ( $n=9-12$ per group; $\left.{ }^{\star} P<0.05\right)$ showing increased body weight and (B) DEXA analysis of body composition ( $n=5-8$ per group; $\left.{ }^{*} P<0.05\right)$ showing increased adiposity in aP2-Dgat1 mice fed a chow or a 16-week high-fat diet. (C) Decreased TG content in the skeletal muscle and livers $(n=9-12$ per group) of aP2-Dgat1 mice fed a high-fat diet ( $\left.{ }^{\star} P<0.05\right)$. (D) Enhanced GTTs (top) and ITTs (bottom) in aP2-Dgat1 mice fed a high-fat diet $\left({ }^{\star} P<0.05\right)(n=5-11$ per group).

However, when fed a 16-week high-fat diet, the weight curves of Adipoq ${ }^{-/-}$mice, which are prone to DIO (39), and Adipoq ${ }^{-/}$aP2-Dgat1 mice were similar (Supplemental Figure 1A), suggesting an epistatic interaction of these alleles in this dietary condition.

As expected, Adipoq ${ }^{-1-}$ mice developed diet-induced glucose intolerance (Supplemental Figure 1B). Remarkably, however, Adipoq ${ }^{-/} \mathrm{aP} 2-D$ gat1 had improved glucose tolerance and lower fasting insulin levels on a high-fat diet than did Adipoq ${ }^{-/}$mice (Supplemental Figure 1B and Supplemental Table 1), indicating that overexpression of DGAT1 in the adipocyte/macrophage compartment was sufficient to suppress the impairment in glucose metabolism induced by adiponectin deficiency. Further, Adipoq ${ }^{-/}$aP2-Dgat1 mice with chronic DIO had lower mRNA levels of M1 macrophage genes in the WAT (Supplemental Figure 1C) and lower serum levels of TNF- $\alpha$, MCP-1, and PAI-1 (Supplemental Figure 1D) than did Adipoq ${ }^{-/-}$mice. TG levels in the serum (Table 1) and in the livers and skeletal muscle (Supplemental Figure 1E) were also lower in Adipoq ${ }^{-/}$aP2-Dgat1 mice with DIO. These results indicate that the protective metabolic effects of DGAT1 overexpression in aP2-Dgat1 mice with DIO are independent of adiponectin.

$B M$ transplantation to generate mice with overexpression of DGAT1 in macrophages. Our studies of aP2-Dgat1 transgenic mice indicated that the overexpression of DGAT1 in the adipocyte/macrophage compartment yielded metabolic benefits in the setting of DIO but could not distinguish the relative contributions of adipocytes and macrophages to this effect. Because of the concomitantly reduced inflammatory state of aP2-Dgat1 mice, we elected to further study the effects of DGAT1 overexpression in monocyte-derived macrophages. To this end, we transplanted lethally irradiated WT mice with BM from age-matched WT or aP2-Dgat1 mice. Our experimental design (Figure 4A) yielded one cohort of mice in which both macrophages and adipocytes were genetically WT $(\mathrm{WT} \rightarrow \mathrm{WT})$ and another in which Dgat1 mRNA levels were increased in macrophages
Table 1

Serum parameters of mice fed a high-fat diet

$\begin{array}{lcccc}\text { Plasma metabolites } & \text { WT } & \text { aP2- } \text { Dgat1 } & \begin{array}{c}\text { Adipoq } \\ \text { FFA }\end{array} & \begin{array}{c}\text { aP2-Dgat1 Adipoq } \\ \text { - }\end{array} \\ \text { TG }(\mathrm{mg} / \mathrm{dl} / \mathrm{l}) & 1.7 \pm 0.1 & 2.0 \pm 0.1^{\mathrm{A}} & 1.87 \pm 0.18 & 1.52 \pm 0.21 \\ \text { TC }(\mathrm{mg} / \mathrm{dl}) & 130.4 \pm 20.5 & 88.6 \pm 11.1^{\mathrm{A}} & 150.0 \pm 28.7 & 98.2 \pm 13^{\mathrm{B}} \\ \text { Glucose }(\mathrm{mg} / \mathrm{dl}) & 154.9 \pm 6.5 & 143.0 \pm 8.9 & 179.9 \pm 8.4 & 146.5 \pm 8.9^{\mathrm{B}} \\ \text { Insulin }(\mathrm{ng} / \mathrm{ml}) & 172.6 \pm 9.3 & 146.1 \pm 10.1^{\mathrm{A}} & 188.0 \pm 5.6 & 187.5 \pm 10.9 \\ \text { HOMA-IR } & 1.24 \pm 0.09 & 1.04 \pm 0.08^{\mathrm{A}} & 2.04 \pm 0.34 & 1.56 \pm 0.31^{\mathrm{B}} \\ \text { Leptin }(\mathrm{ng} / \mathrm{ml}) & 9.4 \pm 0.7 & 6.6 \pm 0.5^{\mathrm{A}} & 16.6 \pm 2.5 & 13.2 \pm 3.1 \\ \text { Resistin }(\mathrm{ng} / \mathrm{ml}) & 10.4 \pm 3.7 & 11.2 \pm 2.1 & 12.4 \pm 2.5 & 14.1 \pm 4.9 \\ \text { Adiponectin }(\mu \mathrm{g} / \mathrm{ml}) & 2.7 \pm 0.5 & 2.9 \pm 0.4 & 3.1 \pm 1.1 & 2.5 \pm 0.4 \\ & 3.4 \pm 0.6 & 5.5 \pm 0.6^{\mathrm{A}} & \mathrm{ND} & \mathrm{ND}\end{array}$

FFA, free FA; TC, total cholesterol; ND, not detectable. $n=7-16$ mice per group. ${ }^{A} P<0.05$ vs WT. ${ }^{\mathrm{B} P}<0.05$ vs Adipoq ${ }^{-1}$. Glucose and insulin measured after 6 -hour fasts. 

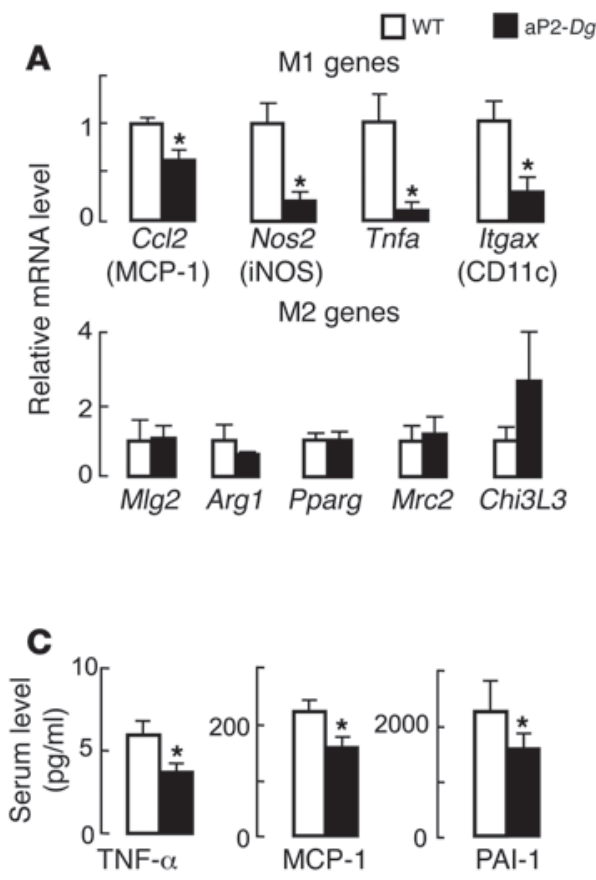

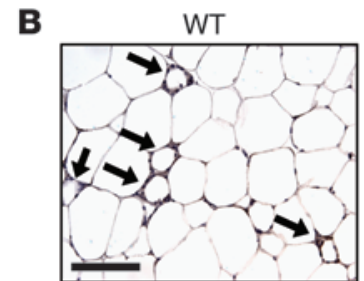

aP2-Dgat1
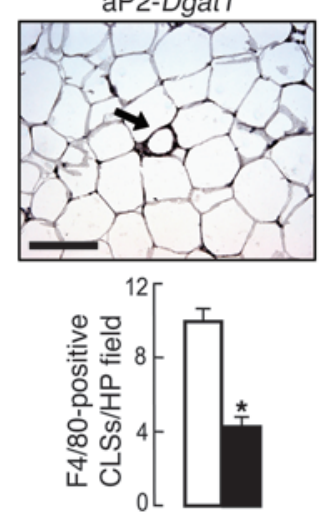

\section{Figure 3}

Reduced markers of WAT and systemic inflammation in aP2-Dgat1 mice chronically fed a high-fat diet. mRNA levels of macrophage M1 and M2 pathway genes were measured by qPCR in WAT of mice fed a high-fat diet for 16 weeks ( $n=7-14$ per group). (A) Decreased mRNA levels of M1 but not M2 genes in WAT of aP2-Dgat1 mice $\left({ }^{*} P<0.05\right)$. M1 genes included iNOS (Nos2), CD11c (Itgax), Tnfa, and MCP-1 (Cc/2). M2 genes included macrophage galactose-/N-acetylgalactosamine-specific lectin (Mg/2), Arginase-1 (Arg1), PPARgamma (Pparg), mannose receptor (Mrc2), and Chitinase3-like 3, or Ym1 (Chi3/3). (B) Fewer macrophage CLS (arrows) in the WAT of aP2-Dgat1 mice fed a high-fat diet ( $n=4-5$ per group). Representative light micrographs of WAT sections stained with antiF4/80 are shown (top), with quantification from serial sections at bottom $\left({ }^{\star} P<0.01\right)$. Scale bar: $50 \mu \mathrm{m}$. (C) Lower levels of serum inflammatory cytokines in serum of aP2-Dgat1 mice fed a high-fat diet $(n=11-14$ per group; ${ }^{*} P<0.05$ ). but not in adipocytes $(\mathrm{Tg} \rightarrow \mathrm{WT})$. By using donor marrow (CD45.2) and recipient marrow $(\mathrm{CD} 45.1)$ that were genetically identical except for a single point mutation, we confirmed that $94 \%-98 \%$ of peripheral leukocytes in transplanted mice were of donor origin 1 month after the procedure (Figure 4B).

To detect aP2-Dgat1 cells in the blood of $\mathrm{Tg} \rightarrow \mathrm{WT}$ mice, we performed PCR on genomic DNA from whole-blood samples with primers unique to the aP2-Dgat1 transgene. The aP2-Dgat1 amplification product was not found in samples from $\mathrm{WT} \rightarrow \mathrm{WT}$ mice but was present in each of the samples from $\mathrm{Tg} \rightarrow \mathrm{WT}$ mice, indicating the reconstitution of blood with aP2-Dgat1 leukocytes in $\mathrm{Tg} \rightarrow \mathrm{WT}$ mice (Figure 4C).

To determine which compartments of the WAT were reconstituted by transplantation, we analyzed Dgat1 mRNA levels in both whole and fractionated WAT. The mRNA levels of the control genes Dgat2 and Emr1 in each WAT compartment were similar between groups (Figure 4D). However, the Dgat1 mRNA levels of Tg $\rightarrow$ WT mice were selectively increased in the SVF and not in the whole WAT or adipocytes (Figure 4D). Taken together, these data are consistent with Tg $\rightarrow$ WT mice having increased Dgat1 mRNA levels in circulating monocytes and WAT macrophages but not in adipocytes.

Increased Dgat1 expression in macrophages is sufficient to protect mice against diet-induced insulin resistance. To determine the metabolic impact of increasing DGAT1 expression in monocyte-derived macrophages, WT $\rightarrow$ WT and $\mathrm{Tg} \rightarrow$ WT mice were fed a high-fat diet for 16 weeks. Both groups of transplanted mice developed a similar level of moderate DIO (Figure 5A). Although this DIO was less marked than that induced in nontransplanted mice (see Figure 2A), both groups of transplanted mice gained weight at a rate greater than that of nontransplanted WT mice fed a chow diet (see Figure 2A) and similar to that in another report of transplanted C57BL/6 mice fed a high-fat diet (43). Unlike aP2-Dgat1 mice, Tg $\rightarrow$ WT mice were not protected against diet-induced nonadipose tissue steatosis (Figure 5B). Remarkably, however, Tg $\rightarrow$ WT mice with DIO exhibited enhanced glucose and insulin tolerance, indicat- ing that the transfer of hematopoietic cells with increased DGAT1 expression is sufficient to protect mice against diet-induced alterations in glucose metabolism (Figure 5C).

Increased Dgat 1 expression in macrophages is sufficient to protect mice against inflammatory consequences of DIO. We next determined whether $\mathrm{Tg} \rightarrow \mathrm{WT}$ mice were protected against the inflammatory consequences of obesity. The mRNA levels of M1 macrophage genes were reduced in the WAT of $\mathrm{Tg} \rightarrow$ WT mice fed a high-fat diet for 20 weeks (Figure 6A), and separation of the WAT revealed a corresponding reduction in the mRNA levels of these genes in the SVF but not in the adipocytes. These findings indicate that transfer of hematopoietic cells with increased Dgat 1 mRNA levels is sufficient to protect mice against diet-induced M1 macrophage activation in the WAT. The mRNA levels of M2 genes were similar in $\mathrm{WT} \rightarrow \mathrm{WT}$ and $\mathrm{Tg} \rightarrow \mathrm{WT}$ mice with chronic DIO, indicating that the protection against macrophage activation in the WAT of Tg $\rightarrow$ WT mice was specific to the M1 pathway (Figure 6B). Consistent with this, WAT sections from $\mathrm{Tg} \rightarrow$ WT mice showed fewer macrophage CLS (Figure 6C), indicating that these mice were protected against the diet-induced accumulation of macrophages in the WAT. The only cytokine with serum levels sufficient for accurate measurement was PAI-1, and these levels were 3 -fold lower in $\mathrm{Tg} \rightarrow \mathrm{WT}$ mice than in WT $\rightarrow$ WT mice (491 \pm 199 versus $1530 \pm 358 \mathrm{pg} / \mathrm{ml} ; n=6-10$ per group; $P<0.05)$.

Dgat1 $m R N A$ levels in cultured macrophages determine TG storage capacity. Our studies in $\mathrm{Tg} \rightarrow$ WT mice showed that increasing DGAT1 expression in macrophages had a profound effect on the M1 activation of WAT macrophages associated with DIO. To determine whether these effects could be extended in vitro, we cultured BMD-M $\phi$ from mice with different levels of DGAT1 expression and treated these cells with monounsaturated oleic acid and saturated palmitic acid, 2 abundant dietary FAs. In particular, treatment of cultured macrophages with palmitate activates the M1 response (12-15).

We first assessed the ability of BMD-M $\phi$ to store FAs as TGs. WT macrophages were incubated with radiolabeled oleate or palmitate for 24 hours to enable examination of FA flux. Both oleate and pal- 
A

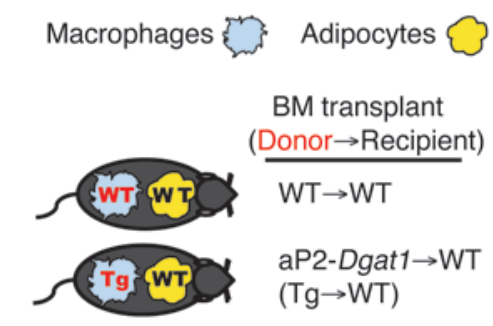

B
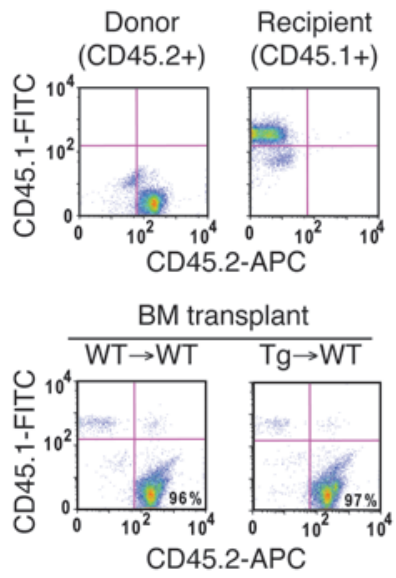

C

Blood

(individual mice)
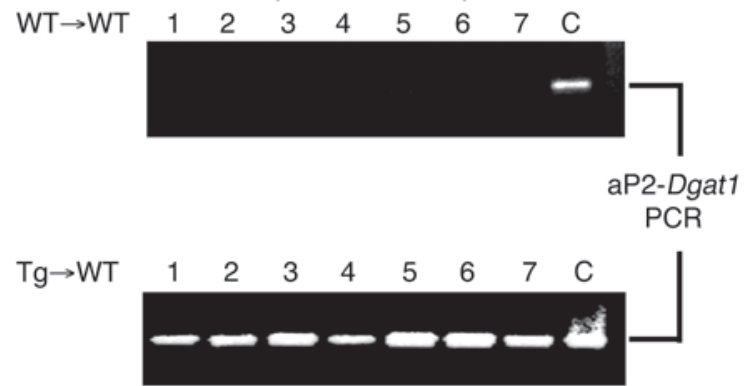

D

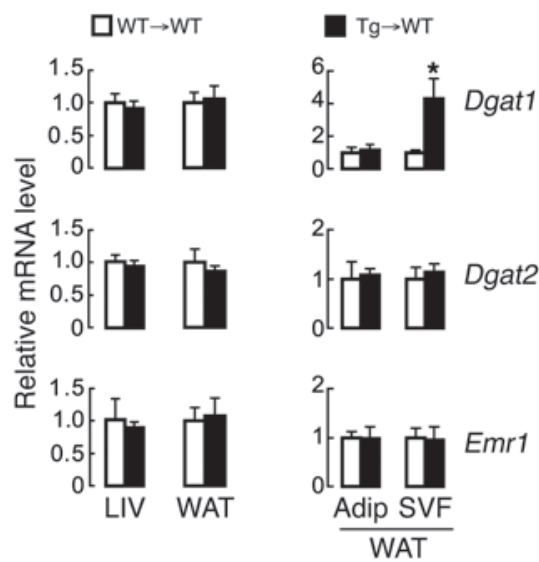

Figure 4

Complete and specific reconstitution in mice transplanted with WT or aP2-Dgat1 BM. (A) Depiction of the Dgat1 genotype expected in adipocytes and macrophages of WT mice transplanted with either WT or aP2-Dgat1 BM. (B) Representative FACS plots of peripheral blood leukocytes from mice, performed with FITC-coupled anti-CD45.1 and APC-coupled anti-CD45.2 antibodies, showing recipient (CD45.1-positive) and donor (CD45.2-positive) cells (top) and that more than 94\% of leukocytes after transplant were of donor origin. (C) Expression of the aP2-Dgat1 transgene is detectable in blood of recipients of Tg $\rightarrow$ WT donors but not WT $\rightarrow$ WT controls. PCR was performed with aP2-Dgat1-specific primers. Tail DNA from known aP2-Dgat1 mice. C indicates a positive control for the transgene. (D) Increased Dgat1 mRNA levels specifically in the SVF of $\mathrm{Tg} \rightarrow$ WT mice. mRNA levels were measured by qPCR from the livers and whole and fractionated WAT of transplanted mice $(n=7-13$ per group) fed a high-fat diet for 20 weeks $\left({ }^{*} P<0.05\right)$.

mitate were taken up by cells and similarly incorporated into the cellular lipid pool (Figure 7A). However, whereas half of the radiolabeled oleate taken up by cells was incorporated into TG, only approximately $12 \%$ of the palmitate was present in this fraction, indicating a preference by WT cells for esterifying oleate (Figure 7A).

We next determined whether changes in Dgat1 mRNA levels altered TG storage capacity in BMD-M $\phi$. Incubation of WT and aP2-Dgat1 BMD-M $\phi$ with oleate showed that macrophages with increased DGAT1 expression had larger and more numerous lipid droplets (Figure 7B). The analysis of BMD-M $\phi$ from Dgat1-deficient (Dgat1 $\left.1^{--}\right)$, WT, and aP2-Dgat1 mice incubated with FAs for 24 hours showed that the level of Dgat1 expression correlated directly with neutral lipid content (Figure 7C). In fact, aP2-Dgat1 BMD-M $\phi$ in medium without added FA (control) had a higher neutral lipid content than WT or Dgat $1^{-/-}$cells. Oleate treatment did not increase the neutral lipid content of $D$ gat $1^{-/-}$cells, but did so for both WT and aP2-Dgat1 cells in a stepwise manner correlating with Dgat1 mRNA level. Furthermore, although palmitate treatment did not increase the neutral lipid content of WT cells (by this method or by TLC) or Dgat1 1-/ cells, it did so for aP2-Dgat1 cells. Thus, the level of Dgat1 mRNA in BMD-M $\phi$ directly correlates with their capacity for TG storage whether treated with oleate or palmitate.
The level of Dgat1 expression modulates M1 inflammatory activation in $B M D-M \phi$. Inasmuch as DGAT1 expression levels affected TG storage capacity in macrophages, we hypothesized that expression levels would also modulate their vulnerability to M1 inflammatory activation in response to FAs. Culturing WT BMD-M $\phi$ with palmitate but not oleate activated the M1 pathway (Figure 8A). We therefore tested to determine whether the level of Dgat 1 mRNA expressed in BMD-M $\phi$ affects the M1 response to palmitate treatment. In untreated (control) cells, the expression of M1 genes was relatively low and varied little between DGAT1 genotypes, although Dgat1 $1^{-/-}$ cells had increased mRNA levels of Nos2 and aP2-Dgat1 cells had decreased levels of Tnfa (Figure 8B). In cells treated with palmitate for 24 hours, mRNA levels of all M1 markers were increased in WT BMD-M $\phi$, and levels of Tnfa and Nos 2 were increased relatively more in $D$ gat $1^{-/-}$cells. In contrast, mRNA levels of all M1 markers were decreased in palmitate-treated aP2-Dgat1 BMD-M $\phi$, indicating an inverse correlation between the levels of Dgat1 mRNA in BMD-M $\phi$ and the induction of $\mathrm{M} 1$ gene expression by palmitate treatment.

M1-type activated macrophages secrete more IL-6, MCP-1, and TNF- $\alpha(1,13,14)$. We therefore measured the levels of these cytokines in the medium of Dgat1 $1^{--}$, WT, and aP2-Dgat1 BMD-M $\phi$ treated with palmitate. In untreated controls, cytokine levels were relatively low, 

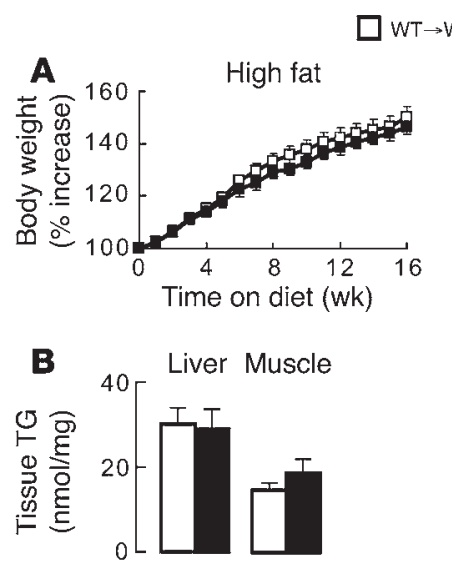

$\mathrm{Tg} \rightarrow W T$

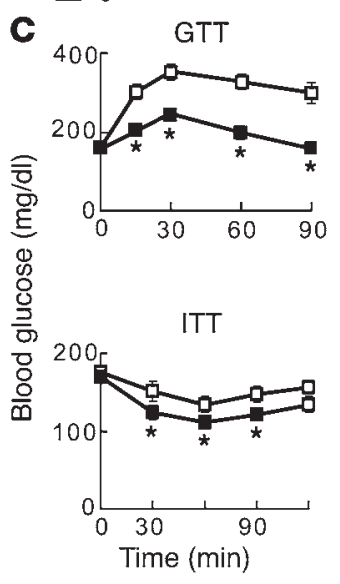

\section{Figure 5}

Transplantation of aP2-Dgat1 BM is sufficient to protect against impaired glucose metabolism in mice chronically fed a high-fat diet. Growth curves (A) and liver and muscle TG content (B) of transplanted mice fed a highfat diet for 20 weeks showing no differences between mice receiving $\mathrm{WT} \rightarrow \mathrm{WT}$ and $\mathrm{Tg} \rightarrow \mathrm{WT}$ mice $(n=7-13$ per group). (C) Enhanced glucose (top, GTT) and insulin (bottom, ITT) tolerance in $\mathrm{Tg} \rightarrow \mathrm{WT}$ mice fed a high-fat diet for 16 weeks $\left({ }^{*} P<0.05\right)$. and medium from $D g a t 1^{-/-}$cells had slightly higher MCP-1 levels than medium from WT and aP2-Dgat1 BMD-M $\phi$ (Figure 8C). Palmitate treatment increased the levels of each cytokine in the medium from BMD-M $\phi$ of each genotype. However, the increase for IL- 6 and TNF- $\alpha$ correlated inversely with the level of Dgat 1 mRNA in the cells, and a similar trend was observed for MCP-1. Thus, increased DGAT1 expression in BMD-M $\phi$ correlated with decreased secretion of proinflammatory substances in response to palmitate treatment.

The effects of DGAT1 expression on macrophage activation were specific for the M1 pathway. The mRNA levels of M2 pathway genes were generally more variable than those of the M1 markers (Supplemental Figure 2). Palmitate treatment did not induce their expression and, in fact, reduced mRNA levels of mannose receptor $(M r c 2)$ in BMD-M $\phi$ of all genotypes. Moreover, the level of DGAT1 expression in these macrophages had no effect on the levels of M2 markers (Supplemental Figure 2).

Interestingly, the effects of increased DGAT1 expression in $\mathrm{BMD}-\mathrm{M} \phi$ were not restricted to M1 activation by FAs. Treatment combining the TLR4 ligand LPS, a canonical M1 stimulator, and IFN- $\gamma$ also increased the mRNA expression of M1 genes in a manner that, in general, correlated inversely with Dgat1 mRNA levels (Supplemental Figure 3). In contrast, the level of DGAT1 expression did not affect the induction of M2 gene expression by treatment with the canonical M2 stimulator IL-4, with the exception of $M r c 2$, in which the effects of IL-4 were mitigated by increased DGAT1 expression (Supplemental Figure 3).

Because the M1 response was reduced in aP2-Dgat1 BMD-M $\phi$ treated with either palmitate or LPS/IFN- $\gamma$, we assessed whether the M1 activation potential of these cells was generally impaired. We treated WT and aP2-Dgat1 BMD-M $\phi$ with acetylated LDL (AcLDL)

\section{Figure 6}

Transplantation of aP2-Dgat1 BM is sufficient to protect mice against diet-induced inflammation in WAT. (A) Lower mRNA levels of M1 pathway genes specifically in the whole WAT and SVF of Tg $\rightarrow$ WT mice. (B) Similar mRNA levels of M2 pathway genes in the whole WAT and SVF of $\mathrm{Tg} \rightarrow \mathrm{WT}$ and WT $\rightarrow$ WT mice. For $\mathbf{A}$ and $\mathbf{B}, \mathrm{mRNA}$ levels were measured by qPCR in whole and fractionated WAT from transplanted mice fed a high-fat diet for 20 weeks $(n=7-13$ per group; $\left.{ }^{*} P<0.05\right)$. (C) Fewer CLS in the WAT of Tg $\rightarrow$ WT mice ( $n=4-5$ mice per group). Macrophages from WAT sections were stained with anti-F4/80 (top), and quantified from serial sections at bottom $\left({ }^{\star} P<0.01\right)$. Scale bars: $50 \mu \mathrm{M}$. and nonopsonized yeast cell-wall $\beta$-glucan (zymosan) particles, which bind to scavenger receptors (44) and dectin-1 (45), respectively, and stimulate M1 macrophage activation. The mRNA levels of M1 genes in WT and AP2-Dgat1 BMD-M $\phi$ were similarly increased by either treatment (Supplemental Figure 4, A and B). Additionally, the phagocytic capacity of aP2-Dgat1 BMD-M $\phi$ activated by zymosan treatment was similar to that of WT cells (Supplemental Figure 4C). Collectively, these results indicate that the M1 activation capacity of aP2-Dgat 1 macrophages is intact in response to other stimuli. We also assessed the M1 activation potential of aP2-Dgat1 macrophages in vivo by inducing peritonitis with thioglycollate treatment. After 3 days, the concentration of F4/80-positive P-M $\phi$ in the peritoneal

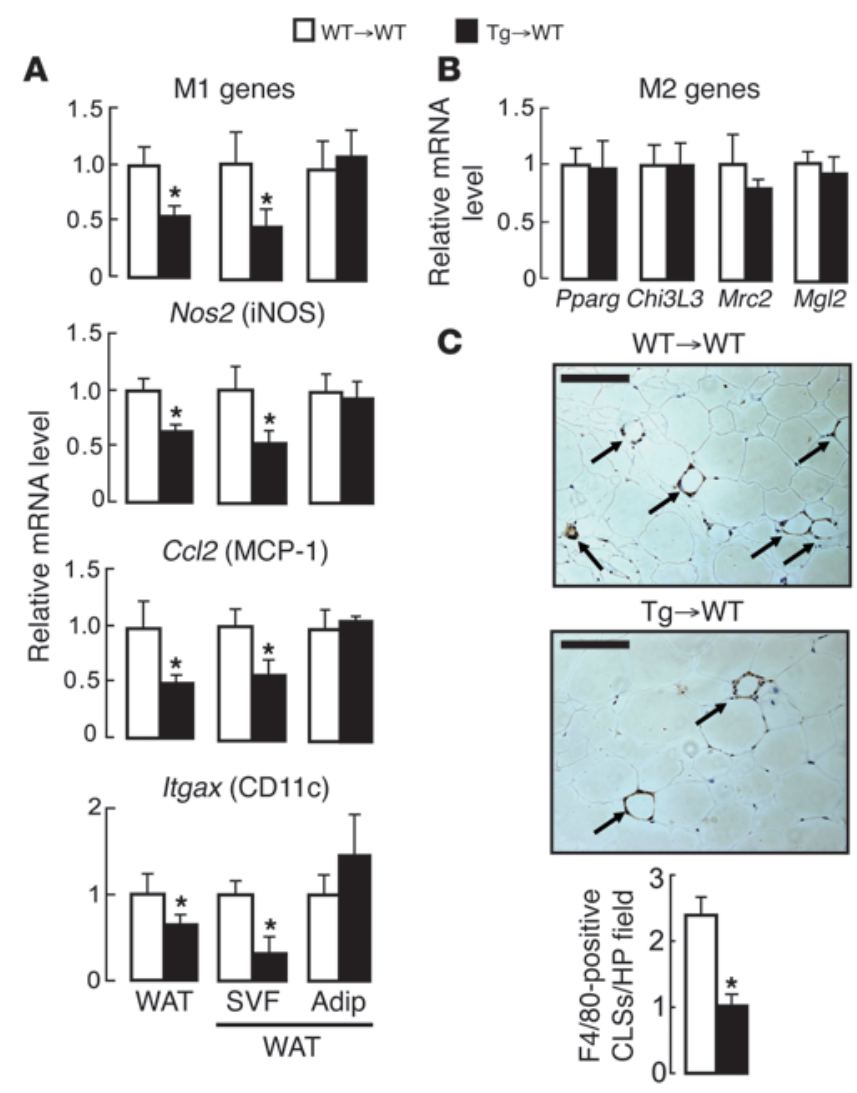


A

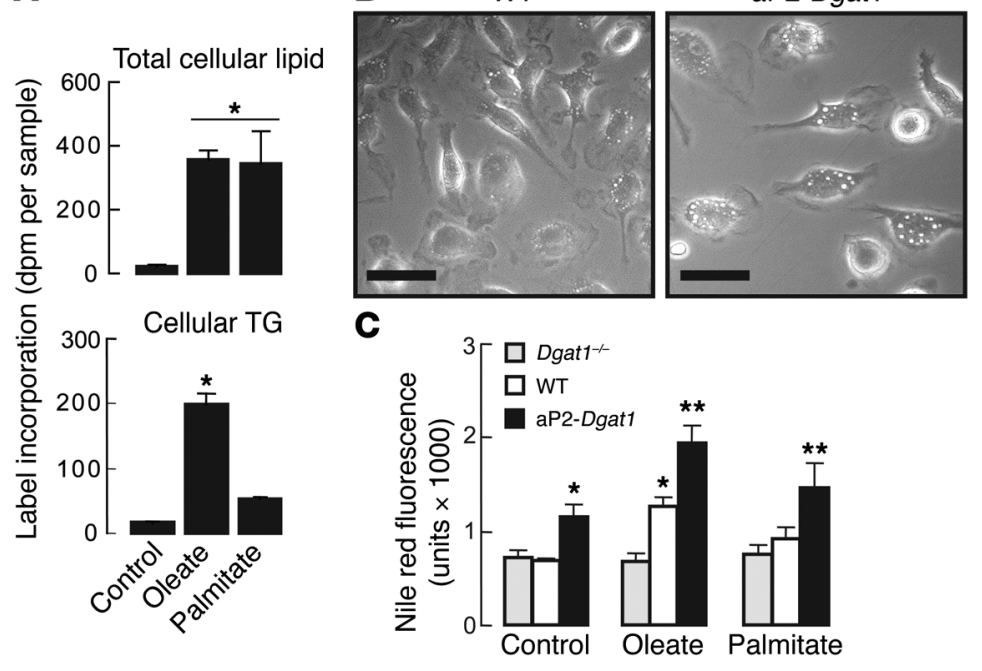

\section{Figure 7}

DGAT1 expression levels correlate with neutral lipid storage in BMD-M $\phi$. (A) WT macrophages take up oleate and palmitate similarly but incorporate more oleate into cellular TG. WT BMD-M $\phi(n=3-4$ per group) were incubated with $100 \mu \mathrm{M}$ of either radiolabeled oleate or palmitate for 24 hours, and dpm were measured from total cellular lipid and the TG fractions after isolation by TLC $\left({ }^{\star} P<0.05\right)$. (B) $\mathrm{BMD}-\mathrm{M} \phi$ from aP2-Dgat1 mice ( $n=3-4$ per group) exhibit more prominent lipid droplets after 24 hours incubation with $200 \mu \mathrm{M}$ oleate. Scale bars: $25 \mu \mathrm{m}$. (C) Neutral lipid content (measured by Nile Red fluorescence) of Dgat $1^{-1-}$, WT, and aP2-Dgat1 BMD-M $\phi$ after 24 hours in medium alone or with $200 \mu \mathrm{M}$ oleate or $150 \mu \mathrm{M}$ palmitate $(n=3-4$ per group; ${ }^{*} P<0.05$ vs WT control, ${ }^{* \star} P<0.05$ versus WT for same treatment condition). fluid was similar in WT and aP2-Dgat1 mice (Supplemental Figure 4D), indicating normal macrophage recruitment, and the mRNA levels of M1 genes were similar in these cells (Supplemental Figure $4 \mathrm{E})$. The mRNA levels of M1 genes were nearly undetectable in peritoneal cells from WT or aP2-Dgat1 mice treated with saline (control). These results indicate that the reduced WAT inflammation seen in aP2-Dgt 1 and $\mathrm{Tg} \rightarrow$ WT mice fed a high-fat diet is not due to a general defect in macrophage recruitment or activation potential.

Dgat1 mRNA levels in BMD-M $\phi$ are increased by PPAR $\gamma$ activation, and DGAT1 is required for PPAR $\gamma$-mediated inhibition of palmitate-stimulated M1 activation. PPAR $\gamma$ agonists are potent antidiabetic agents that can suppress the M1 activation of cultured macrophages by palmitate treatment (13). Additionally, DGAT1 expression is increased by PPAR $\gamma$ agonist treatment in WAT and in cultured adipocytes (28-30). By extrapolation, we sought to determine whether PPAR $\gamma$ agonists regulate DGAT1 expression in macrophages and whether DGAT1 plays a role in PPAR $\gamma$-mediated inhibition of M1 activation. Treatment of WT BMD-M $\phi$ with the PPAR $\gamma$ agonists pioglitazone $(10 \mu \mathrm{M})$ or troglitazone $(10$ or $100 \mu \mathrm{M})$ increased Dgat1 mRNA levels approximately 2-fold, whereas treatment with $10 \mu \mathrm{M}$ WY14643, a PPAR $\alpha$ agonist, had no effect (Figure 9A). We next treated WT or Dgat $1^{-/-}$BMD-M $\phi$ with either palmitate $(150 \mu \mathrm{M})$ alone for 24 hours or with pioglitazone $(10 \mu \mathrm{M})$ for 24 hours followed by palmitate and pioglitazone together for another 24 hours. As expected, the mRNA levels of M1 genes were increased by palmitate treatment, and this effect was inhibited by pre- and cotreatment with pioglitazone (Figure 9B). In contrast, pioglitazone treatment was unable to suppress the M1 activation in Dgat1 1-- BMD-M $\phi$ (Figure 9B). A similar pattern was found for MCP-1, IL-6, and TNF- $\alpha$ levels in the secreted medium; these levels were increased by palmitate treatment and reduced by PPAR $\gamma$ agonists in WT cells, but the pioglitazone effect was absent in Dgat1-/cells (Figure 9C). These data indicate that DGAT1 is required for the protective effects of pioglitazone in suppressing the M1 response of macrophages treated with saturated FAs.

\section{Discussion}

We demonstrate here that increased expression of the TG synthesis gene Dgat 1 in macrophages is sufficient to protect against the inflammatory and metabolic consequences of DIO in vivo and against M1 inflammatory activation of macrophages treated with FAs or cytokines in vitro. aP2-Dgat1 transgenic mice, which had increased Dgat1 mRNA levels in the adipocyte/macrophage compartment, were prone to DIO but protected against DIO-associated nonadipose tissue steatosis, WAT and systemic inflammation, and insulin resistance. Remarkably, the protection against dietinduced WAT inflammation and insulin resistance in aP2-Dgat1 mice could be conferred to WT mice by the simple transfer of hematopoietic cells with increased DGAT1 expression, highlighting the importance of this enzyme within the macrophage compartment in preventing inflammation associated with excess lipids. In corroboration, in vitro studies of macrophages showed that Dgat 1 mRNA levels correlated directly with macrophage TG storage capacity and inversely and specifically with M1 macrophage activation by palmitate. Further, Dgat1 mRNA levels in macrophages were regulated by PPAR $\gamma$ activation, and DGAT1 was required for PPAR $\gamma$ inhibition of the M1 response to palmitate treatment. These findings identify DGAT1 as an important modulator of lipid-induced inflammation in macrophages.

A finding of this study that we believe to be novel is that DGAT1 overexpression in the adipocyte/macrophage compartment of transgenic mice increased their propensity to DIO but markedly protected them from the associated deleterious metabolic and inflammatory consequences. In a prior study of the same aP2-Dgat1 mice, we showed that the aP2-Dgat1 transgene was expressed in the WAT and found that these mice were prone to DIO on a short-term (4 weeks) high-fat diet (24). In that study, we showed that glucose tolerance was similar in WT and aP2-Dgat1 mice in response to this short-term diet. In the current study, we show that these mice are protected from developing insulin resistance in response to a chronically high-fat diet (16 weeks). Moreover, this protection extended to include pronounced reductions in M1-type inflammation in the WAT and nonadipose tissue steatosis. The mRNA levels of several M1 inflammatory genes, including $\mathrm{Ccl} 2$, which encodes the macrophage-secreted chemokine MCP-1, and Tnfa, which encodes the inflammatory cytokine TNF- $\alpha$, were decreased in the WAT of aP2-Dgat1 mice, and levels of inflammatory cytokines were lower in the circulation of aP2-Dgat1 mice with DIO. By crossing these mice with Adipo ${ }^{-1-}$ mice, we showed that this phenotype was not dependent on the presence 
A
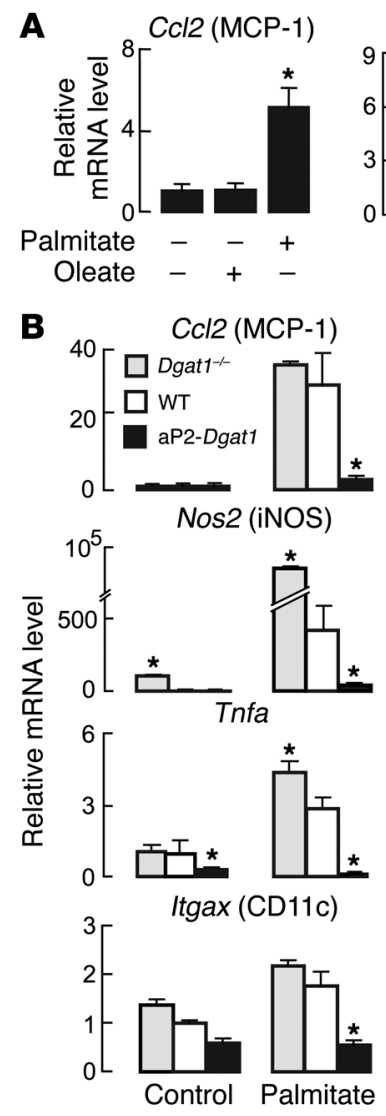
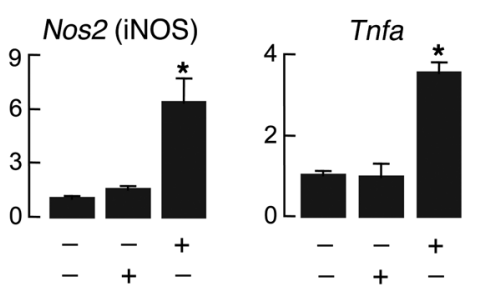

C
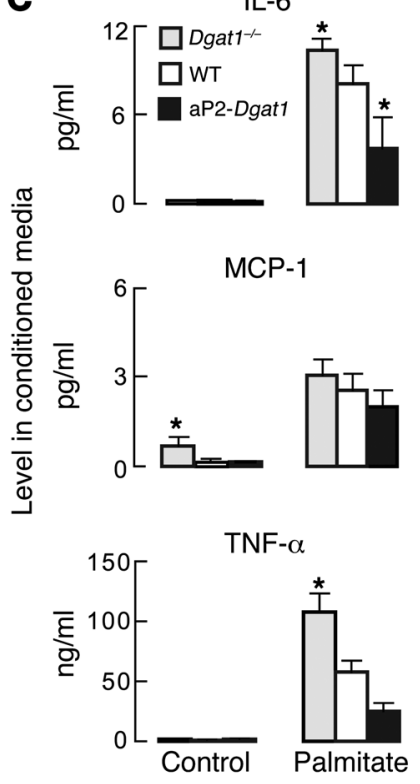

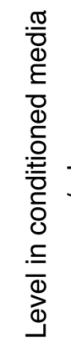

of adiponectin, a hormone that is secreted by adipocytes and promotes insulin sensitivity $(29,37-40)$, thus suggesting alternative mechanisms, such as macrophage expression of the transgene.

A second major finding of this study is that increasing DGAT1 expression in macrophages by the transfer of hematopoietic cells was sufficient to protect mice from the deleterious consequences of DIO. Remarkably, BM transplantation of hematopoietic cells was sufficient to recapitulate the enhanced glucose metabolism and antiinflammatory aspects of aP2-Dgat 1 mice. On the other hand, increasing DGAT1 expression in macrophages was insufficient to alter body weight or prevent diet-induced hepatic or skeletal muscle steatosis, indicating that the effects were specific for the insulin-sensitizing and antiinflammatory components of the aP2-Dgat1 phenotype and did not apply to TG partitioning. Of note, that $\mathrm{Tg} \rightarrow$ WT mice experienced metabolic protection without a relative reduction in liver TG suggests that hepatic steatosis per se is unlikely to contribute significantly to the development of insulin resistance in this model.

Our findings in the BM transplantation studies do not discount the possibility that increased TG storage capacity in adipocytes may also contribute to the phenotype of aP2-Dgat1 mice. Indeed, enhanced capacity for FA storage in adipocytes may serve to limit the exposure of macrophages to proinflammatory FAs. Additionally, recent studies have shown that adipocytes can regulate macrophage activity $(14,46,47)$. Studies focusing on adipocyte-specific DGAT1 expression in regulating the inflammatory and metabolic consequences of DIO are needed to address this question.

The phenotype in our 2 studies of aP2-Dgat1 mice contrasts with a report of a different aP2-Dgat1 transgenic line that exhibited glucose intolerance and tissue steatosis in response to a high-fat diet (48).

\section{Figure 8}

DGAT1 expression levels modulate M1-type activation of BMD-M $\phi$ by palmitate. (A) Treatment with palmitate but not oleate increases M1 gene mRNA levels in WT BMD-M $\phi$. mRNA levels were measured by qPCR ( $n=3-6$ per group; $\left.{ }^{*} P<0.05\right)$. (B) Expression of M1 pathway genes from Dgat1--, WT, and aP2-Dgat1 BMD-M $\phi$ in medium alone (control) or after treatment for 24 hours with $150 \mu \mathrm{M}$ palmitate $(n=3-7$ per group; ${ }^{*} P<0.05$ vs WT in the same treatment condition). (C) Levels of IL-6, MCP-1, and TNF- $\alpha$ in conditioned medium of control and palmitate-treated BMD-M $\phi\left(n=6-7\right.$ per group; ${ }^{*} P<0.05$ vs WT).

While we do not know for certain what accounts for this discrepancy, our current study suggests that one possible contributing variable might be the amount of DGAT1 expression in macrophages, which was not measured in the other model. If those mice, in contrast to ours, have little aP2-driven DGAT1 expression in macrophages, then they may have been more prone to WAT inflammation and insulin resistance in response to a high-fat diet. Other variables that may contribute to the different phenotypes include genetic background (the other model was on an FVB background, whereas our model was on $\mathrm{C} 57 \mathrm{BL} / 6$ ) or the timing or duration of the high-fat diet employed. The other mice were studied after 6 weeks of a high-fat diet starting at the age of 24-29 weeks, whereas we studied the effects of high-fat diets of at least 16 weeks starting at age 12 weeks.

Our BM transplantation studies suggested that macrophages with increased DGAT1 and increased lipid synthesis capacity might be protected from the proinflammatory effects of lipids. Indeed, we found that macrophages isolated from mice that overexpress DGAT1 had an increased capacity for TG storage and were resistant to the M1 inflammatory response that results from treatment with palmitate. Conversely, macrophages lacking DGAT1 were more sensitive to palmitate-stimulated M1 activation. These findings are consistent with DGAT1 having a role in protecting cells from lipotoxicity mediated by FAs, as was shown in other cell types (27). In the case of macrophages within the WAT, this function for DGAT1 may be of considerable importance, since these cells may encounter and take up large amounts of lipids by endocytic or phagocytic means.

Precisely how DGAT1 protects against M1 macrophage activation by FAs is unclear. We found that Dgat $1 \mathrm{mRNA}$ levels correlated inversely with the level of M1 activation in macrophages treated with palmitate or the combination of LPS (a TLR4 ligand) and IFN- $\gamma$. Interestingly, this correlation was not seen when M1 activation was stimulated in WT and aP2-Dgat1 macrophages by treatment with AcLDL, a ligand for scavenger receptors (44), or nonopsonized zymosan, a ligand for dectin-1 (45). Taken together, these findings suggest that palmitate and LPS/IFN- $\gamma$ may signal through a shared downstream step modulated by DGAT1. This finding is consistent with other work suggesting that saturated FAs and LPS engage common signaling elements, including JNK1, JNK2, NF- $\mathrm{B}$, and activating transcription factor 3 , an NF- $\mathrm{B}$ target $(13-17,49)$.

FAs may also regulate macrophage activation by mechanisms distinct from that of LPS. In support of this, the kinetics by which LPS treatment upregulates Tnfa mRNA levels in RAW 264.7 macrophages were quite different from those induced by palmitate treatment (14). While LPS acts extracellularly, our findings support DGAT1 functioning to modulate FA flux intracellularly. Increased esterification of intracellular FAs into the TG pool may act as a "sink" to lower their flux into pathways affecting the levels of signaling metabolites linked to inflammation (ceramides, 
A

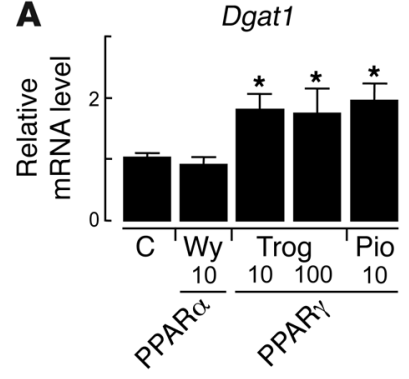

B

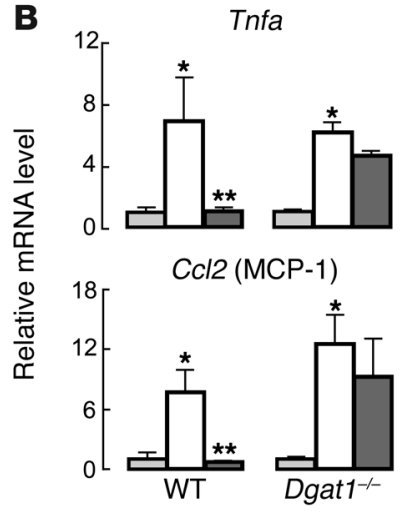

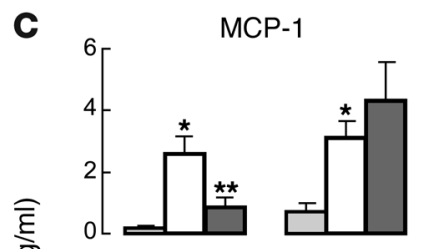

IL-6

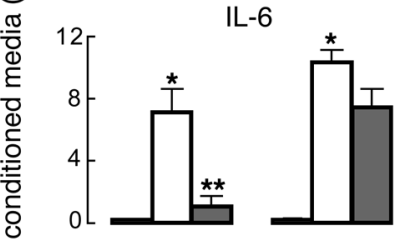

TNF- $\alpha$

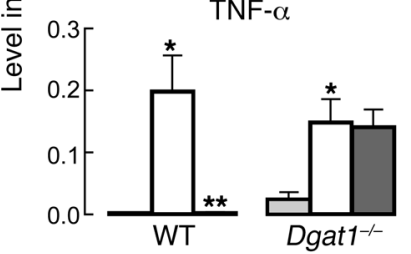

$\square$ Control

$\square$ Palmitate

Palmitate + Pio

eicosanoids, diacylglycerols, lysophosphatidic acids) and ER stress, which itself can induce JNK phosphorylation and NF-KB activation in cells $(20-22,50-53)$.

In contrast to the effects on the M1 pathway, the level of Dgat1 mRNA expression in macrophages had little effect on IL-4-mediated activation of the $\mathrm{M} 2$ response, further highlighting the specificity of the DGAT1 effect on inflammatory pathways. We note, however, that a more appropriate analysis of the consequences of altering DGAT1 expression on the M2 pathway in macrophages would require studying mice on the BALB/c genetic background, which exhibit a more robust M2 macrophage phenotype than do those on the C57BL/ 6 background in the current study (54).

By sensing levels of intracellular FA metabolites, PPAR $\gamma$ links nutritional status to the transcriptional regulation of metabolic genes. In macrophages, PPAR $\gamma$ activity regulates the expression of M1 and M2 genes $(19,31-33,55)$ and modulates FA-induced inflammatory activation (13). Since PPAR $\gamma$ activation increases Dgat 1 mRNA levels in adipocytes (28-30), we hypothesized that a similar pathway may exist in macrophages. Indeed, mRNA levels of Dgat1 were increased by pharmacologic activation of PPAR $\gamma$ in cultured macrophages. More importantly, DGAT1 was required for the antiinflammatory effects of PPAR $\gamma$ in suppressing FA-mediated M1 inflammatory activation. This suggests that DGAT1 is an important mediator of the beneficial effects of PPAR $\gamma$ agonists in this cell type. Chronic DIO in humans, as in mice, is associated with WAT inflammation (5), and the systemic manifestation of this inflammation is ameliorated by treatment with PPAR $\gamma$ agonists (33). Our findings suggest that the level of DGAT1 expression in human macrophages is a determinant of this process and that DGAT1 may be involved in the antiinflammatory effects of PPAR $\gamma$.

We note that the mRNA levels of both murine Dgat1 and Dgat2 were markedly increased in WT P-M $\phi$ in response to chronic highfat feeding. This pattern likely reflects an adaptive change in the gene expression program of macrophages to facilitate the esterifi-

\section{Figure 9}

Dgat1 mRNA levels are increased by PPAR $\gamma$ agonists in macrophages, and DGAT1 is necessary for protective effects of PPAR $\gamma$ activation in macrophages treated with saturated FAs. (A) Dgat1 mRNA levels are increased by PPAR $\gamma$ agonists in cultured BMD-M $\phi$ ( $n=3-4$ per group). $B M D-M \phi$ were incubated in medium alone (control) or treated for 24 hours with PPAR $\alpha$ agonist (10 $\mu \mathrm{M}$ WY 14643) or PPAR $\gamma$ agonists (10 or $100 \mu \mathrm{M}$ troglitazone [Trog] or $10 \mu \mathrm{M}$ pioglitazone [Pio]) as indicated $\left({ }^{*} P<0.05\right)$. (B) PPAR $\gamma$ activation suppresses M1 response to palmitate treatment in macrophages of WT but not Dgat $1^{-1-}$ mice. mRNA levels in BMD-M $\phi(n=6-7$ per group) incubated in medium alone (control) or with either $150 \mu \mathrm{M}$ palmitate or the combination of $150 \mu \mathrm{M}$ palmitate and $10 \mu \mathrm{M}$ pioglitazone were measured by qPCR ( ${ }^{\star} P<0.05$ vs control, ${ }^{* *} P<0.05$ vs palmitate). (C) PPAR $\gamma$ activation suppresses cytokine secretion from WT but not Dgat1-/- BMD-M $\phi$ treated with palmitate $\left({ }^{\star} P<0.05\right.$ vs control; ${ }^{\star \star} P<0.05$ vs palmitate).

cation of high levels of FAs and their derivatives. The mechanism for these changes in DGAT gene expression in macrophages is unknown but offers an opportunity for further investigation.

The current findings raise an interesting paradox when compared with those from DGAT1-knockout mice, which also exhibit increased insulin sensitivity (56) and reduced markers of inflammation in the WAT (R. Streeper, S. Cases, and R. Farese, unpublished observations). Thus, whole-body DGAT1 deficiency is associated with reduced WAT inflammation, yet DGAT1 deficiency in isolated macrophages increased the propensity to FA-mediated inflammation. What could account for this? We suspect that whole-body DGAT1 deficiency effectively prevents FA accumulation in the WAT, through shunting of FA to oxidative and thermogenic pathways and through a decreased rate at which dietary FAs enter the circulation (57). In such a model, DGAT1-deficient macrophages would not be exposed to toxic levels of FAs that would normally induce inflammation. Thus, the overall phenotypic effects of DGAT1 deficiency may depend on the relative contributions of different cell types. This implication may have clinical relevance, since highly potent and specific inhibitors of DGAT1 activity are being developed to treat clinical obesity and related TG-associated disease (58).

\section{Methods}

Mice. Studies were of WT C57BL/6J and genetically modified mice, including aP2-Dgat1 transgenic (24), Dgat1 $1^{-/-}$(56), and Adipoq ${ }^{-/-}$mice (39). aP2-Dgat1, Dgat $1^{-/-}$, and Adipo ${ }^{-/-}$mice were on a C57BL/6J background (backcrossed for more than 10 generations). Pups were weaned onto a standard 5053 PicoLab chow diet (Purina). For DIO studies, 12-week-old mice were fed a Western-type diet containing $20 \%$ anhydrous milk fat, $1 \%$ corn oil, and $0.2 \%$ cholesterol by weight (TD.01064; Harlan-Teklad) for 16 weeks. For BM transplantations, donor mice were C57BL/6J (CD45.2; Jackson Laboratory), and recipients were B6.SJL-Ptprc ${ }^{a} P e p 3^{b} /$ BoyJ (CD45.1; Taconic). Anesthesia was induced with isoflurane (survival procedures) or with avertin or halothane (terminal procedures). All procedures were approved by the UCSF Animal Research Committee and followed NIH guidelines.

Isolation of BMD-M $\phi$. Marrow from the femurs and tibias of 22- to 26week-old mice fed a chow or a chronic high-fat diet was collected by flushing with $10 \mathrm{ml}$ of RPMI (Gibco; Invitrogen) containing 20\% heat-inactivated FBS (Hyclone). The suspension was passed through a $70-\mu \mathrm{m}$ cell strainer, incubated in red cell lysis buffer for 10 minutes at room temperature, washed twice, and resuspended in RPMI with FBS. Cells were plated at a density of 1 to $2 \times 10^{6}$ per well in medium supplemented with $30 \%$ L929-conditioned medium to stimulate differentiation of precursors into 
BMD-M $\phi$. Noncoated tissue culture plates were used to prevent adherence of dendritic and nonprogenitor cells. The medium was changed every 3 days; after 6 days, nearly all cells had differentiated into macrophages by visual analysis. Cells were harvested 8 to 12 days after plating.

Isolation of primary $P-M \phi$. The peritoneal cavities of mice fed a chow or chronic high-fat diet were infused with $10 \mathrm{ml}$ of ice-cold PBS, and after agitation, approximately $7 \mathrm{ml}$ of fluid recovered from each mouse was processed and plated as described for BM samples. Cells were washed 3 hours after plating to isolate adherent $\mathrm{P}-\mathrm{M} \phi$ and cultured for 3 days, after which the medium was changed. P-M $\phi$ were used 4 days after plating.

$B M$ transplantation. To facilitate the assessment of BM reconstitution, the genetic background of the recipient mice (B6.SJL-Ptprc ${ }^{a}$ Pep $3^{b}$ /BoyJ) was identical to that of the donor mice (C57BL/6), except for a single point mutation in the leukocyte antigen Ptprc (CD45.2). The mutant version of Ptprc (CD45.1) is functionally equivalent, but allows the identification of cells that are of recipient (CD45.1) or donor (CD45.2) origin in peripheral blood leukocytes by FACS (see below). BM cells harvested from 8-week-old WT or aP2-Dgat1 donor mice were resuspended in PBS with $0.1 \%$ FA-free BSA, and 1 to $1.2 \times 10^{6}$ donor cells were injected into age- and sex-matched recipient mice by tail vein. Recipient mice were irradiated in two 5-Gy doses spaced 3 hours apart 1 day before injection. After BM injections, recipient mice were placed in their home cages with antibiotic water for 1 month, after which blood was drawn for analysis of BM reconstitution by FACS and for detection of aP2-Dgat1 leukocytes with transgene-specific primers. The mice then recovered under usual care until they were 12 weeks old, at which time they were placed on a high-fat diet. Glucose tolerance tests (GTTs) and insulin tolerance tests (ITTs) were performed as for other cohorts of mice after 16 weeks of diet, and tissues were harvested after 20 weeks of diet.

FACS analyses. The percentage of CD $45.1^{+}$(recipient) and CD 45.2 $2^{+}$ (donor) cells in the peripheral blood of BM-transplanted mice was determined by flow cytometry (FACSCalibur; BD Biosciences). Data were acquired with CellQuest Pro software (version 5.1.1; BD Biosciences) and analyzed using FlowJo software (version 6.4.7; Tree Star). Gating was set using the Zebra plot feature of FlowJo, based on unstained samples, and CD $45.1^{+}$and CD45.2 $2^{+}$cells were identified using FITC-coupled antiCD45.1 (1:200) and allophycocyanin-coupled (APC-coupled) anti-CD45.2 (1:1000) antibodies, respectively (BD - Pharmingen). The percentage of donor cells was expressed by multiplying the total number of live cells by the percentage of CD $45.2^{+}$cells.

To measure acute macrophage recruitment, peritoneal fluid was obtained from mice 3 days after intraperitoneal injection with $1 \mathrm{ml}$ of $1 \%$ thioglycollate (BD Biosciences) to induce transient aseptic peritonitis. The percentage of macrophages in this fluid was determined by flow cytometry as above, and macrophages were identified using a PE-conjugated antiF4/80 antibody (1:1000; Caltag Laboratories). The concentration of P-M $\phi$ was expressed by multiplying the total number of live cells in the peritoneal fluid by the percentage of $\mathrm{F} 4 / 80^{+}$cells.

Separation of SVF and adipocyte fractions of WAT. Epididymal fat pads were excised from adult chow-fed mice and placed in HBSS (Gibco; Invitrogen) containing $1 \%$ HEPES and 3\% BSA. Fat pads were finely minced, washed twice in DMEM/F-12 (Gibco; Invitrogen), and placed in DMEM/F-12 containing $1 \mathrm{mg} / \mathrm{ml}$ collagenase (type I; Worthington) at $37^{\circ} \mathrm{C}$ for 30 minutes with gentle agitation. The cell suspension was filtered through a $250-\mu \mathrm{m}$ nylon filter (Nitex; Safar America), and the filtrate was spun at $80 \mathrm{~g}$ for 5 minutes at room temperature to separate the pellet containing the SVF from the floating adipocytes. The adipocytes were resuspended in medium containing $5 \mathrm{mM}$ EDTA and 2\% BSA in PBS and spun again at $200 \mathrm{~g}$ for 5 minutes. Floating cells were plated. SVF cells were spun again at $600 \mathrm{~g}$, and the pelleted cells were plated as for PM- $\phi$.
Real-time PCR. RNA was isolated using RNA Stat-60 (Tel-Test), according to the manufacturer's protocol, from homogenized whole epididymal WAT, separated WAT fractions, or cultured macrophages. RNA $(0.5-2 \mu \mathrm{g})$ was reverse-transcribed with SuperScript III reverse transcriptase and random hexamers (Invitrogen) to generate cDNA. Real-time qPCR was performed with an ABI Prism 7700 (Applied Biosystems) and SYBR green detection of amplified products. Each $25-\mu \mathrm{l}$ PCR reaction mix contained $2 \mu \mathrm{l}$ of cDNA, $12.5 \mu \mathrm{l}$ of SYBR green master mix (QIAGEN), and $10 \mathrm{pmol}$ of each primer. Relative mRNA abundance was normalized to the internal standard cyclophilin with the $\Delta \Delta C T$ method, as described by the manufacturer (Invitrogen). Primer sequences are listed in Supplemental Table 1.

Immunoblotting. The abundance of DGAT1 protein was detected in BMD-M $\phi$ by immunoblotting. Briefly, confluent cells in 6-well plates were partially dislodged by incubating them at $37^{\circ} \mathrm{C}$ in PBS with $10 \mathrm{mM}$ EDTA for 10 minutes. The cells were then washed twice, manually scraped into $200 \mu \mathrm{l}$ of PBS, and spun at $800 \mathrm{~g}$ for 5 minutes. The supernatant was discarded, and the cells were resuspended in $500 \mu \mathrm{l}$ of buffer containing $50 \mathrm{mM}$ Tris ( $\mathrm{pH}$ 7.4) and $250 \mathrm{mM}$ sucrose. The suspended cells were passed several times through a 27 -gauge needle, then spun at $15,000 \mathrm{~g}(\max )$ for 5 minutes at $4^{\circ} \mathrm{C}$. Protein $(10 \mu \mathrm{g})$ from the supernatant of each sample was loaded onto a $10 \%$ Bis-Tris gel (NuPAGE; Invitrogen), and SDS-PAGE was performed using compound 3 -(N-morpholino) propanesulfonic acid (MOPS) as the running buffer. Proteins were transferred to PVDF membranes over 90 minutes, and membranes were blocked at $4{ }^{\circ} \mathrm{C}$ overnight in $0.1 \%$ Tween- 20 containing $5 \%$ nonfat milk (TBS-T). Membranes were then washed and incubated in TBS-T containing 1:2000 anti-DGAT1 antibody (polyclonal rabbit anti-mouse) for 2 hours, washed again, and incubated for another 2 hours in TBS-T containing 1:2000 HRP-conjugated anti-rabbit IgG (ECL; GE Healthcare). Protein abundance was detected by chemiluminescence (ECL-plus). As an internal loading control, membranes were stripped and reprobed with murine monoclonal anti$\beta$-actin antibody (1:2500; Sigma-Aldrich) for 45 minutes and incubated in TBS-T containing HRP-conjugated anti-mouse IgG for 1 hour prior to detection of chemiluminescence (Super Signal West Pico; Thermo Scientific).

Measurement of tissue TG content. Liver and skeletal muscle (gastrocnemius and soleus) samples were pulverized in liquid nitrogen and homogenized in buffer A (50 mM Tris- $\mathrm{HCl}, \mathrm{pH} 7.4,250 \mathrm{mM}$ sucrose) containing proteinase inhibitors. Lipids were extracted in chloroform:methanol (2:1) and separated by TLC on silica gel G-60 plates with the solvent hexane: ethyl ether:acetic acid (80:20:1). The TG bands were visualized by exposure to iodine and then scraped and analyzed as described (59), with triolein (Sigma-Aldrich) as a standard, and expressed per tissue weight.

Measuring incorporation of FAs into cellular TG. FA fluxes were followed in $\mathrm{BMD}-\mathrm{M} \phi$ treated with medium containing oleate or palmitate. Each FA was a mixture of cold $(100 \mu \mathrm{M})$ and ${ }^{14} \mathrm{C}$-labeled $(0.04 \mu \mathrm{Ci} / \mathrm{ml})$ forms, with the label appearing at the distal carbon. Cells were incubated for 24 hours, after which total lipid was extracted from cell lysates and separated by TLC to visualize the TG fraction as above. FA incorporation was then determined by scintillation counting of ${ }^{14} \mathrm{C}$ from both total cellular lipid and the cellular TG fraction, respectively.

Nile Red fluorescence assay. BMD-M $\phi$ cultured in 12-well plates (BD Falcon) were incubated in DMEM plus FBS alone or in medium containing either $200 \mu \mathrm{M}$ oleate or $150 \mu \mathrm{M}$ palmitate (both from Sigma-Aldrich). Oleate and palmitate were prepared and complexed with BSA (2:1 molar ratio of FA to albumin) as described (27). After 24 hours, intracellular neutral lipid content was measured by Nile Red fluorescence with the AdipoRed assay reagent as described by the manufacturer (Cambrex). Fluorescence was measured with a Victor ${ }^{3} 1420$ fluorometer (PerkinElmer) at excitation and emission wavelengths of $485 \mathrm{~nm}$ and $572 \mathrm{~nm}$, respectively. To correct for nonspecific fluorescence, measurements taken before incubation with Nile Red were subtracted from those taken after incubation. 
Activation of $M 1$ and M2 inflammatory pathways in BMD-M $\phi$. Cells were incubated in medium alone (control), for 24 hours in medium containing

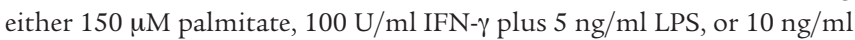
IL-4 (all from Sigma-Aldrich), for 6 hours in medium containing $50 \mu \mathrm{g} / \mathrm{ml}$ AcLDL (gift from Tobias Walther, Max Planck Institute of Biochemistry, Martinsried, Germany), or for 2 hours in medium containing zymosan at a particles/cells ratio of approximately 50:1 (Cell BioLabs). Cells were then lysed for RNA isolation, and the medium was collected for cytokine measurements. The concentration and duration of palmitate treatment have been shown to be nontoxic (12), and we did not observe any signs of toxicity in the cells we treated. Activation of PPAR $\gamma$ in BMD-M $\phi$ was stimulated by incubating cells with troglitazone (Sigma-Aldrich) or pioglitazone (gift from Brian Hubbard). Activation of PPAR $\alpha$ was stimulated by incubating cells with WY14643 (Sigma-Aldrich).

Phagocytosis assay. Phagocytosis of zymosan particles by BM-M $\phi$ was measured colorimetrically at $405 \mathrm{~nm}$ using a kit (Cell BioLabs) per manufacturer instructions.

Immunohistochemistry. Formalin-fixed, paraffin-embedded epididymal WAT sections were deparaffinized, rehydrated, and treated with antigen-unmasking reagent, as described by the manufacturer (Vector Laboratories). Sections were blocked in normal serum and incubated with biotinylated anti-F4/80 antibody (Serotec) overnight. Endogenous HRP activity was quenched by incubation with $3 \%$ hydrogen peroxide for 10 minutes. Samples were then incubated with streptavidin-HRP (BD Biosciences), and specific HRP activity was detected with 3,3'-diaminobenzidine (DAB; Vector Laboratories). Sections were counterstained with hematoxylin before dehydration and mounting. CLS were counted from 10 separate $\times 20$ fields per sample in serial samples, and the mean numbers of CLS per sample in each group were plotted.

Analyses of serum and medium. Mice were fasted for 12 hours (overnight) for lipid analyses and 8 hours for hormone levels. Blood was obtained from tail veins of unanesthetized mice, and kits were used to measure the levels of free FA (Wako Pure Chemical Industries), TG (Roche Diagnostics), and total cholesterol (Wako). Levels of insulin, adiponectin, and resistin in serum and levels of inflammatory markers (MCP-1, IL-6, PAI-1, and TNF- $\alpha$ ) in both serum and culture medium were measured by multiplexed bead-based immunoassays (Millipore Corporation) using xMAP technology (Luminex). Blood glucose of mice fasted for 8 hours was measured with a glucometer (OneTouch Ultra; LifeScan).

Assessment of glucose and insulin tolerance and insulin sensitivity in vivo. Glucose tolerance was assessed in mice after an 8-hour fast by measuring basal blood glucose and monitoring glucose excursion $15,30,60,90$, and 120 minutes after a peritoneal injection of dextrose $(1 \mathrm{~g} / \mathrm{kg})$. Insulin tolerance was assessed in mice after a 2 -hour fast by measuring basal blood glucose and its excursion after an injection of insulin $(1 \mathrm{U} / \mathrm{kg})$. Insulin sensitivity was expressed as an index (HOMA-IR) calculated as follows: $(\mathrm{G} 0 \times \mathrm{I0}) / 22.5$, where $\mathrm{G}_{0}$ is fasting glucose $(\mathrm{mmol} / \mathrm{l})$, and $\mathrm{I0}$ is insulin $(\mu \mathrm{U} / \mathrm{ml})(60)$.

Body composition. Body composition in mice fasted for 4 hours was analyzed under isoflurane anesthesia by DEXA with a PIXImus2 scanner (GE Healthcare Lunar).

Statistics. Data are presented as the mean \pm SEM. Measurements were compared by 2-tailed Student's $t$ test or Mann-Whitney rank-sum test. Body weights were compared by repeated-measures ANOVA with Bonferroni's post-hoc analysis. GTTs and ITTs were analyzed similarly and also by comparing the areas under each curve calculated by the trapezoid rule (Prism4; GraphPad Software). Significance was determined at $P<0.05$.

\section{Acknowledgments}

We thank S. Coughlin for facilitating BM transplantation experiments, C. Mrejen for assistance with TLC analyses in macrophages, Y. Si for assistance with FACS of blood samples, K. Veenstra and S. Marmor for performing immunoassays, J.D. Fish for histological assistance, R. Bituin for mouse husbandry, G. Howard for editorial assistance, D. Jones for manuscript preparation, and members of the Farese laboratory for discussions and comments. This work was supported by grants from the NIH (DK056084 to R.V. Farese Jr.; DK08174-01 to S.K. Koliwad; animal facilities grant NIH/NCRR CO6 RR018928; and the UCSF Diabetes, Endocrinology and Metabolism Training Grant NIDDK DK07418-27 to S.K. Koliwad) and the A.P. Giannini Foundation for Medical Research (to S.K. Koliwad), and by the J. David Gladstone Institutes.

Received for publication August 27, 2009, and accepted in revised form December 9, 2009.

Address correspondence to: Suneil K. Koliwad or Robert V. Farese Jr., Gladstone Institute of Cardiovascular Disease, 1650 Owens Street, San Francisco, CA 94158. Phone: 415.734.2718; Fax: 415.355.0960; E-mail: skoliwad@gladstone.ucsf.edu (S.K. Koliwad); bfarese@gladstone.ucsf.edu (R.V. Farese Jr.).
1. Lumeng CN, Bodzin JL, Saltiel AR. Obesity induces a phenotypic switch in adipose tissue macrophage polarization. J Clin Invest. 2007;117(1):175-184.

2. $\mathrm{Xu} \mathrm{H}$, et al. Chronic inflammation in fat plays a crucial role in the development of obesity-related insulin resistance. J Clin Invest. 2003;112(12):1821-1830.

3. Wellen K, Hotamisligil G. Obesity-induced inflammatory changes in adipose tissue. J Clin Invest. 2003;112(12):1785-1788.

4. Cinti S, et al. Adipocyte death defines macrophage localization and function in adipose tissue of obese mice and humans. J Lipid Res. 2005;46(11):2347-2355.

5. Weisberg SP, et al. Obesity is associated with macrophage accumulation in adipose tissue. J Clin Invest. 2003;112(12):1796-1808.

6. Hotamisligil GS. Inflammation and metabolic disorders. Nature. 2006;444(7121):860-867.

7. Sartipy P, Loskutoff D. Monocyte chemoattractant protein 1 in obesity and insulin resistance. Proc Natl Acad Sci U S A. 2003;100(12):7265-7270.

8. Shoelson S, Lee J, Goldfine A. Inflammation and insulin resistance. J Clin Invest. 2006;116(7):1793-1801.

9. Linton M, Fazio S. Macrophages, inflammation, and atherosclerosis. Int J Obes Relat Metab Disord. 2003;27(Suppl 3):S35-S40.

10. Boden $\mathrm{G}$. Interaction between free fatty acids and glucose metabolism. Curr Opin Clin Nutr Metab Care. 2002;5(5):545-549.

11. Kim J. Fat uses a TOLL-road to connect inflammation and diabetes. Cell Metab. 2006;4(6):417-419.

12. Laine PS, et al. Palmitic acid induces IP-10 expression in human macrophages via NF-kap$\mathrm{paB}$ activation. Biochem Biophys Res Commun. 2007;358(1):150-155.

13. Nguyen MT, et al. A subpopulation of macrophages infiltrates hypertrophic adipose tissue and is activated by free fatty acids via toll-like receptors 2 and 4 and JNK-dependent pathways.J Biol Chem. 2007;282(48):35279-35292.

14. Suganami T, et al. Role of the Toll-like receptor 4/NF-kappaB pathway in saturated fatty acidinduced inflammatory changes in the interaction between adipocytes and macrophages. Arterioscler Thromb Vasc Biol. 2007;27(1):84-91.

15. Shi H, Mokoeva MV, Inouye K, Tzameie I, Yin $\mathrm{H}$, Flier JS. TLR4 links innate immunity and fatty acid-induced insulin resistance. $J$ Clin Invest. 2006;116(11):3015-3025.
16. Hirosumi J, et al. A central role for JNK in obesity and insulin resistance. Nature. 2002;420(6913):333-336.

17. Lee JY, et al. Saturated fatty acid activates but polyunsaturated fatty acid inhibits Toll-like receptor 2 dimerized with Toll-like receptor 6 or 1.J Biol Chem. 2004;279(17):16971-16979.

18. Xu H, et al. Molecular recognition of fatty acids by peroxisome proliferator-activated receptors. $\mathrm{Mol}$ Cell. 1999;3(3):397-403.

19. Ricote M, Li AC, Willson TM, Kelly CJ, Glass CK. The peroxisome proliferator-activated receptor- $\gamma$ is a negative regulator of macrophage activation. Nature. 1998;391(6662):79-82.

20. Martins de Lima T, et al. Mechanisms by which fatty acids regulate leucocyte function. Clin Sci. 2007;113(2):65-77.

21. Fueller M, Wang D, Tigyi G, Siess W. Activation of human monocytic cells by lysophosphatidic acid and sphingosine-1-phosphate. Cell Signal. 2003;15(4):367-375.

22. Hammarstrom $S$, et al. Novel eicosanoid activators of PPAR gamma formed by RAW 264.7 macrophage cultures. Adv Exp Med Biol. 2002;507:343-349.

23. Yen CL, Stone SJ, Koliwad S, Harris C, Farese RV Jr. Thematic review series: glycerolipids. DGAT 
enzymes and triacylglycerol biosynthesis. J Lipid Res. 2008;49(11):2283-2301.

24. Chen HC, Stone SJ, Zhou P, Buhman KK, Farese RV Jr. Dissociation of obesity and impaired glucose disposal in mice overexpressing acyl coenzyme a: diacylglycerol acyltransferase 1 in white adipose tissue. Diabetes. 2002;51(11):3189-3195.

25. Levin MC, et al. Increased lipid accumulation and insulin resistance in transgenic mice expressing DGAT2 in glycolytic (type II) muscle. Am J Physiol Endocrinol Metab. 2007;293(6):E1772-E1781.

26. Monetti M, et al. Dissociation of hepatic steatosis and insulin resistance in mice overexpressing DGAT in the liver. Cell Metab. 2007;6(1):69-78.

27. Listenberger LL, et al. Triglyceride accumulation protects against fatty acid-induced lipotoxicity. Proc Natl Acad Sci U S A. 2003;100(6):3077-3082.

28. Ruan H, Pownall H, Lodish H. Troglitazone antagonizes tumor necrosis factor-alpha-induced reprogramming of adipocyte gene expression by inhibiting the transcriptional regulatory functions of NF-kappaB. J Biol Chem. 2003;278(30):28181-28192.

29. Kim JY, et al. Obesity-associated improvements in metabolic profile through expansion of adipose tissue. J Clin Invest. 2007;117(9):2621-2637.

30. Ranganathan $G$, et al. The lipogenic enzymes DGAT1, FAS, and LPL in adipose tissue: effects of obesity, insulin resistance, and TZD treatment. J Lipid Res. 2006;47(11):2444-2450.

31. Hevener A, et al. Macrophage PPAR gamma is required for normal skeletal muscle and hepatic insulin sensitivity and full antidiabetic effects of thiazolidinediones. J Clin Invest. 2007;117(6):1658-1669.

32. Odegaard JI, et al. Macrophage-specific PPARgamma controls alternative activation and improves insulin resistance. Nature. 2007;447(7148):1116-1120.

33. Mohanty P, et al. Evidence for a potent anti-inflammatory effect of rosiglitazone. J Clin Endocrinol Metab. 2004;89(6):2728-2735.

34. Makowski L, et al. Lack of macrophage fatty-acidbinding protein aP2 protects mice deficient in apolipoprotein E against atherosclerosis. Nat Med. 2001;7(6):699-705.

35. Rolph MS, et al. Regulation of dendritic cell function and $\mathrm{T}$ cell priming by the fatty acid-binding protein aP2. J Immunol. 2006;177(11):7794-7801.

36. Steppan CM, et al. The hormone resistin links obe- sity to diabetes. Nature. 2001;409(6818):307-312.

37. Pajvani $U$, et al. Complex distribution, not absolute amount of adiponectin, correlates with thiazolidinedione-mediated improvement in insulin sensitivity. J Biol Chem. 2004;279(13):12152-12162.

38. Maeda $N$, et al. Diet-induced insulin resistance in mice lacking adiponectin/ACRP30. Nat Med. 2002;8(7):731-737.

39. Streeper RS, Koliwad SK, Villanueva CJ, Farese RV Jr. Effects of DGAT1 deficiency on energy and glucose metabolism are independent of adiponectin. Am J Physiol Endocrinol Metab. 2006; 291(2):E388-E394.

40. Yamauchi $\mathrm{T}$, et al. The fat-derived hormone adiponectin reverses insulin resistance associated with both lipoatrophy and obesity. Nat Med. 2001;7(8):941-946.

41. Okamoto Y, et al. Adiponectin inhibits the production of CXC receptor 3 chemokine ligands in macrophages and reduces T-lymphocyte recruitment in atherogenesis. Circ Res. 2008;102(2):218-225.

42. Takemura Y, et al. Adiponectin modulates inflammatory reactions via calreticulin receptor-dependent clearance of early apoptotic bodies. J Clin Invest. 2007;117(2):375-386.

43. Furuhashi M, Fucho R, Görgün CZ, Tuncman G, Cao H, Hotamisligil GS. Adipocyte/macrophage fatty acid-binding proteins contribute to metabolic deterioration through actions in both macrophages and adipocytes in mice. J Clin Invest. 2008;118(7):2640-2650.

44. Krieger M, Herz J. Structures and functions of multiligand lipoprotein receptors: Macrophage scavenger receptors and LDL receptor-related protein (LRP). Ann Rev Biochem. 1994;63:601-637.

45. Brown GO, Gordon S. Immune recognition. A new receptor for beta-glucans. Nature. 2001;413(6851):36-37.

46. Li Y, et al. Extracellular Nampt promotes macrophage survival via a nonenzymatic interleukin-6/STAT3 signaling mechanism. J Biol Chem. 2008;283(50):34833-34843

47. Kang K, et al. Adipocyte-derived Th2 cytokines and myeloid PPARdelta regulate macrophage polarization and insulin sensitivity. Cell Metab. 2008;7(6):485-495

48. Chen N, Liu L, Zhang Y, Ginsberg HN, Yu YH.
Whole-body insulin resistance in the absence of obesity in FVB mice with overexpression of Dgat1 in adipose tissue. Diabetes. 2005;54(12):3379-3386.

49. Suganami T, et al. Activating transcription factor 3 constitutes a negative feedback mechanism that attenuates saturated Fatty acid/toll-like receptor 4 signaling and macrophage activation in obese adipose tissue. Circ Res. 2009;105(1):25-32.

50 . Boradaile NM, et al. A critical role for eukaryotic elongation factor 1A-1 in lipotoxic cell death. Mol Biol Cell. 2006;17(2):770-778.

51. Cunha DA, et al. Initiation and execution of lipotoxic ER stress in pancreatic beta-cells. J Cell Sci. 2008;121(Pt 14):2308-2318.

52. Guo W, Wong S, Xie W, Lei T, Luo Z. Palmitate modulates intracellular signaling, induces endoplasmic reticulum stress, and causes apoptosis in mouse 3T3-L1 and rat primary preadipocytes. AmJ Physiol Endocrinol Metab. 2007;293(2):E576-E586.

53. Ron D, Walter P. Signal integration in the endoplasmic reticulum unfolded protein response. Nat Rev Mol Cell Biol. 2007;8(7):519-529.

54. Mills CD, Kincaid K, Alt JM, Heilman MJ, Hill AM. M-1/M-2 macrophages and the Th1/Th2 paradigm. JImmunol. 2000;164(12):6166-6173.

55. Pascual G, et al. A SUMOylation-dependent pathway mediates transrepression of inflammatory response genes by PPAR-gamma. Nature. 2005;437(7059):759-763

56. Chen HC, et al. Increased insulin and leptin sensitivity in mice lacking acyl CoA:diacylglycerol acyltransferase 1.J Clin Invest. 2002;109(8):1049-1055.

57 . Buhman KK, et al. DGAT1 is not essential for intestinal triacylglycerol absorption or chylomicron synthesis. J Biol Chem. 2002;277(28):25474-25479.

58. Zhao G, et al. Validation of diacyl glycerolacyltransferase I as a novel target for the treatment of obesity and dyslipidemia using a potent and selective small molecule inhibitor. J Med Chem. 2008;51(3):380-383.

59. Snyder F, Stephens NA. A simplified spectrophotometric determination of ester groups in lipids. Biochim Biophys Acta. 1959;34:244-245.

60. Lee $S$, et al. Comparison between surrogate indexes of insulin sensitivity and resistance and hyperinsulinemic euglycemic clamp estimates in mice. $A m J$ Physiol Endocrinol Metab. 2008;294(2):E261-E270. 